\title{
Article
}

\section{Decision making and risk management in adventure sports coaching}

Collins, L., and Collins, D.

Available at http://clok.uclan.ac.uk/12421/

Collins, L., ORCID: 0000-0002-7478-1140 and Collins, D. ORCID: 0000-00027601-0454 (2013) Decision making and risk management in adventure sports coaching. Quest, 65 (1). pp. 72-82. ISSN 0033-6297

It is advisable to refer to the publisher's version if you intend to cite from the work. http://dx.doi.org/10.1080/00336297.2012.727373

For more information about UCLan's research in this area go to

http://www.uclan.ac.uk/researchgroups/ and search for < name of research Group>.

For information about Research generally at UCLan please go to http://www.uclan.ac.uk/research/

All outputs in CLoK are protected by Intellectual Property Rights law, including Copyright law. Copyright, IPR and Moral Rights for the works on this site are retained by the individual authors and/or other copyright owners. Terms and conditions for use of this material are defined in the policies page.

\section{CLoK}

Central Lancashire online Knowledge www.clok.uclan.ac.uk 
This is a pre-proof corrected manuscript, as accepted for publication, of an article published by Taylor \& Francis in Quest on $16^{\text {th }}$ January 2013, available online:

http://www.tandfonline.com/doi/abs/10.1080/00336297.2012.727373

\section{PLEASE REFER TO THE PUBLISHED VERSION FOR CITING PURPOSES}

Decision Making and Risk Management in Adventure Sports Coaching

Loel Collins* \& Dave Collins

Institute of Coaching and Performance, School of Sport, Tourism and the Outdoors, University of Central Lancashire, Preston, United Kingdom

*Correspondence concerning this paper should be directed to Loel Collins, Greenbank Building, Institute of Coaching and Performance, University of Central Lancashire, Preston, PR1 2HE, United Kingdom. Email: LCollins2@uclan.ac.uk 


\section{Introduction}

Risk management is an essential element of a coach's skill set (Ferrero, 2007) and, reflecting this central role, the topic plays a small but significant part in the vast majority of coach education programs (cf. British Canoe Union, 2007). For the outdoor professional, however, risk management is a more subtle and wider ranging skill (Collins \& Collins, 2012). This distinction presents challenges for the Adventure Sports Coach (ASC) who must optimally manage the inherent risks associated with Adventure Sports (AS), such as rock climbing, mountaineering, sea kayaking, white water kayaking, canoeing and caving, against the longer term developmental needs and shorter term stated goals of clients.

All AS are characterized by a degree of risk, since they require specific technical skills, possess an element of physical challenge and occur in a continually changing, (largely) noncompetitive and dynamic environment. One key characteristic of this challenge is that risk plays a central role in adventure, has a synergetic relationship with the challenge and is often a major factor in participation. Indeed, participants may seek out the input from the ASC to allow them to better their own experience and develop skills that support their high pressure, 'in the field' decision making. In this regard, Brevik (2007, p. 11) states, "we should confront danger and take calculated risks, but only when we have developed the necessary skills and experience" thereby illustrating the dilemma that, in developing the skills, learners will almost inevitably experience danger and risk. Based on this observation, the appropriate preparation of the ASC is even more crucial to ensuring a safe, effective and enjoyable experience for participants.

In response to this need, Collins and Collins (2012) attempt to conceptualize the role of the ASC, proposing that the broad range of motivations for participation in AS creates the parallel need for a broad skill-set for the ASC. The ASC requiring a pedagogic skill set that encompasses coaching, leadership and personal development skills in order to respond to the differentiated needs of a client group. Furthermore, the nature of AS generates a need for a personal ability in the activity itself that underpins these professional skills, this does not require the ASC to be a high performer but certainly to be skillfully independent in the teaching and adventure environment. These components, coaching, leadership, development and personal ability, must also be integrated with highly developed judgment 
and decision-making skills. In short, the major characteristics required of the ASC seem best described as both diverse and integrated while operating synergistically. These characteristics are summarized in Figure 1.

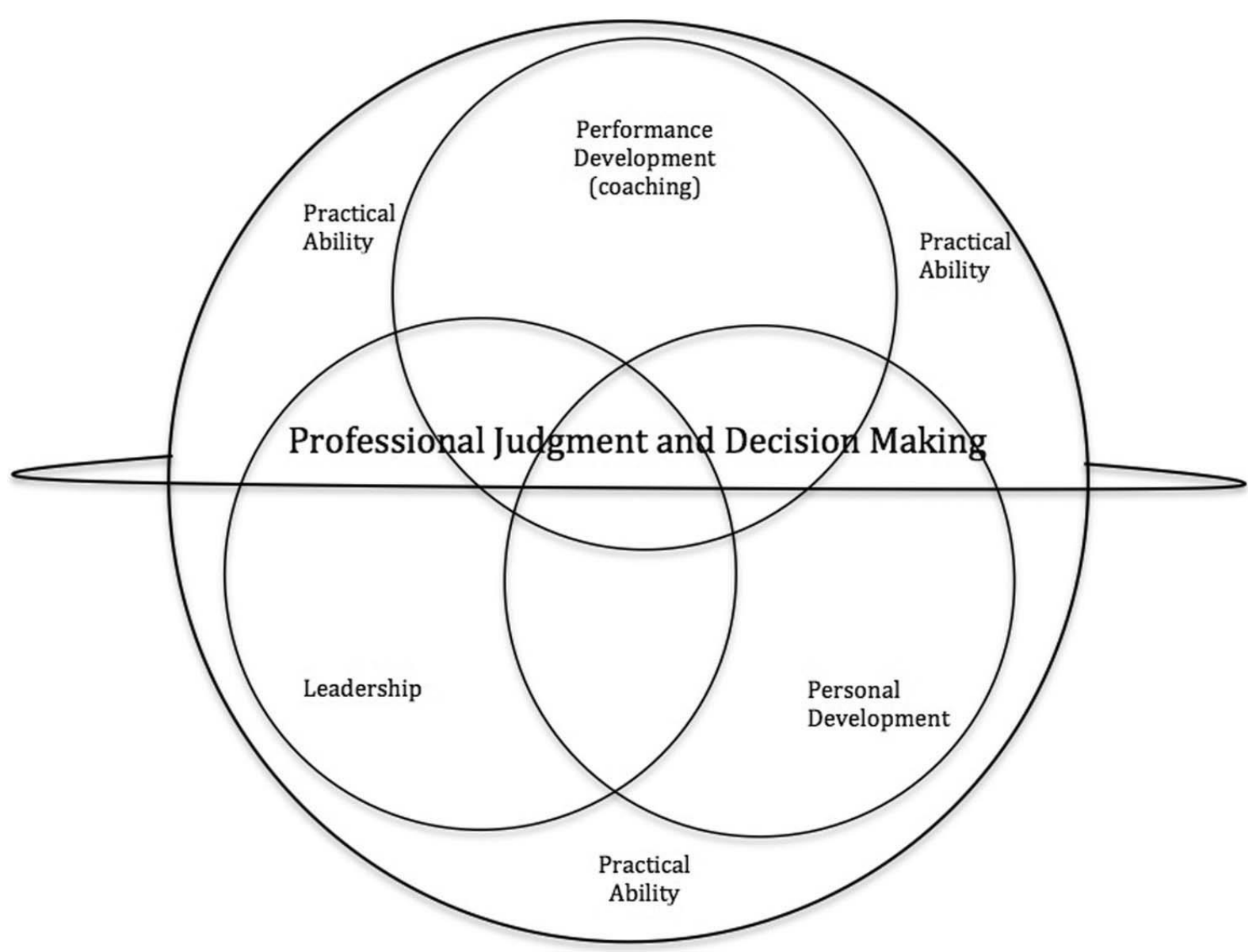

Figure 1. Conceptualization of the adventure sports coach, adapted from Collins \& Collins (2012).

\section{Different Approaches to Risk Management}

Clearly, the ASC must deploy a careful and considered approach to risk management, balancing the challenge of an activity with the potential benefits, while maintaining a level of risk that is authentic for the learner. In contrast, perhaps, to other sports, risk is perceived as a central component of outdoor education (Brown \& Fraser, 2009; Wurdinger, 1997), one that must be exploited rather than minimized. (Priest \& Gass, 2005) recognize this as the 'central paradox' for 
adventure experiences. The 'traditional' approaches to risk assessment typically employed by other sports, such as the Health and Safety Executive's 'five steps to risk assessment' (2006), namely hazard identification, identification of those at risk, risk evaluation and control, recording of actions and review of assessment, unfortunately, frequently lacks the adaptability necessary to accommodate the dynamic environments encountered in adventure activities. This 'schizophrenia' between the need to control risk and simultaneously utilize it is a significant challenge for the practicing ASC.

When addressing these issues, risk-benefit analysis (Ball, Gill, \& Spiegel, 2008; Gill, 2010) is seen as a logical and essential progression from the simpler risk elimination approaches which characterize the methods taught in most coach education programs in other more traditional sports and, unfortunately, still in many ASC programs. Risk benefit analysis is increasingly common in the UK, both in education and AS environments. For example, Priest and Gass (2005, p. 93) state, "You need a clear understanding of the benefits of risk taking in order to determine whether these benefits outweigh the risk." Indeed, Young (2010) recognizes the practice in a broader setting and advocates that all educational risk management should be based around risk benefit analysis. In such an approach, coaches, teachers and leaders across sports would generate all assessments built around a 'justification' of stated risks against the benefits shown to flow from them. Debate will remain on a society's wider acceptance that there is value in risk taking, although ultimately this may align with the professional AS community's acceptance of the educational benefit.

Thus, against this backdrop, and in contrast to the history and culture of 'minimizing' risk, the issue is now one of focusing on and understanding the benefit in order to exploit it, rather than just an emphasis on negative implications alone. As reflected earlier, the overall benefit of exposure to risk during human development is increasingly being recognized (Tovey, 2007) and an understanding that challenge is essential in a healthy and developing society (Moxnes, 1989) has produced a shift in thinking. Csikszentmihalyi (1990) suggests that this 'risky edge,' the feeling of being stretched, can stimulate learning behavior. Changes may also, in part, reflect a growing trend against the 'compensation culture"1 (Young, 2010) and a broader acceptance that personal responsibility cannot be abdicated in a modern society. 
Further, however, we suggest that risk-benefit analysis should be extended when used by the ASC so as to produce an approach in which risks are still identified 'up front,' but which are then immediately considered against their potential for exploitation rather than elimination or justification: an additional or even distinct complication that requires the perception of risk as both a positive and negative factor in the decision making process (Chambers, Odeggard, \& Rinaldi, 2007; McDaniels \& Small, 2004). Of course, this enhanced risk benefit analysis approach assumes that the ASC is capable of detecting, understanding and empathizing with the individual participant's perceptions and understanding of risk, and is able to integrate this with the risks presented by the environment. Brown and Fraser (2009) highlight the complexities associated with decision making in this manner, an additional challenge that almost inevitably requires some differences in the process of coach planning, in event actions and in preparation. Whatever the underlying causes, however, there is an increasing acknowledgement that risk benefit is a more effective and parsimonious method than the five steps model, at least for ASCs. However to apply this approach effectively a clear understanding of the potential benefits will be required.

\section{Understanding the Benefits in Risk Benefit Analysis}

Traditionally, the benefits of adventure, outdoor, and experiential educative approaches have been difficult to quantify, especially from a personal development perspective. Different authors identify a range of benefits that encompass the short, medium and long term. Unsurprisingly perhaps, these often appear to relate to the specified learning outcomes of the program undertaken (Hattie, Marsh, Neill, \& Richards, 1997; Gassner \& Russel, 2008). While supporting this approach, Hunt (1990) highlights the difficulty in quantifying the educational benefit and suggests that discussions frequently focus on learning outcomes as a way of defining this gain. However these are operationalized, their nature is often of a higher order; they are skills, attitudes and competencies, for example which are proposed or even expected to transfer from the adventure setting into 'normal' life (cf., Hattie et al., 1997; Gassner \& Russel, 2008). For the educationalist this lack of clarity remains a complex challenge.

Of course, for the ASC benefit may be more easily quantified at a lower and more 'immediate' level, in terms of improved performance. A clear mental model of an 'ideal' performance 
(e.g., what the new skill will look like) enables the coach to make a qualitative assessment based on observation, questioning and improvement, thereby 'justifying' the exposure to risk. Even here, however, the more complex and hard to define psychological benefits, such as confidence and focus, also play a part since they contribute to the level of performance that may be achieved.

Whichever way the benefits are defined and conceptualized, however, this must form a central part of the pre-planning structure and in-event action with regard to risk management. Hunt (1990) suggests that this is an 'ethical problem' which may be addressed by quantifying risk and benefit into independent sets of numbers that could be compared, a solution also proposed by Wilson and Crouch (2001). However, Hunt (1990) also acknowledges that this approach does not reflect the complexity of risk and the variety of the environments that characterize AS, implicitly acknowledging the complexity of the decision making process which underpins the risk benefit trade-off. Risk is frequently understood in negative terms; Lupton and Tulloch (2002) claiming it is synonymous with danger and hazard, whereas Ward (2008) links risk to unacceptable threats that can be physical, financial, psychological, or social. Crucially, however, Ward also identifies that risk is not only a negative impact or threat but it can also be positive or beneficial (cf. Moxnes, 1989).

By contrast, the risk benefit analysis advocated by Ball et al. (2008) is a descriptive, nonformulaic approach that, in itself, may have inherent problematic issues; most notably, that of qualifying the potential benefit of the activity. Ball et al.'s model is based on the premise that 'experts' can make sound judgments about risks and benefits in their fields of expertise. In an AS context this may have to be explored further, as an inconsistent mental model of performance may make analysis on this 'personal perception' basis potentially flawed.

Equally, the personal nature of learning, motivation and risk perception will also impact on this perceptual process: namely, one participant benefits from a learning experience may well be different from the benefit experienced by another. Although such inter-individual contrasts may be desirable and reflect differentiated learning requirements, they make it very difficult to accurately identify all the benefits for all the participant's. Furthermore, each participant's perception of risk will vary; both generically based on their personality and previous experience, and dynamically through the activity based on an interaction of these factors with aspects of the environment, including the 
coach's behavior. These individual concerns just further complicate the already difficult issue of managing risk/benefit.

Perceptions of risk may be influenced by many factors (Breakwell, 2007; Wilson \& Crouch, 2001), not least the individual's interpretation of their own experience (Breakwell, 2007). ASCs create their perceptions in response to extensive experience over a period of time, which clients through necessity do so from a more limited experience, however. This mismatch has the potential for a further conflict between the desired impact of an experience designed by the ASC and the actual impact on the student. For example, Brymer and Gray (2010) suggested that the younger demographic of participants may be the 'thrill seekers' while older participants more commonly seek out interaction with the environment as a primary orientation. Older ASCs may have different perceptions of management of risk from those of younger participants or coaches (Breakwell, 2007). Perceptions of risk, benefit, and impact on the learner will directly inform the utilization of risk in practice. The decision making process by the ASC on how to optimize the experience of learning by exploiting risk will be complex.

\section{Exploiting the Benefits: Decision Making in Relation to Risk}

Cross (1999), Lyle and Cushion (2010), and Martin, Cashel, Wagstaff, and Breunig (2006) all suggest that the collection of information is fundamental in coach decision making. They propose that effective decision-making involves creating events and changing the future while also acknowledging that a lack of action may also lead to change. This logically suggests that the decision on whether to make a decision or not will, in itself, lead to other related decisions in dynamic environments. In simple terms, even doing nothing is changing something! This concept of parallel agendas, or nested thinking (Abraham \& Collins, 2011), has a direct relevance in the risk management process for ASCs, in that decisions will be not only be taken against immediate challenges (such as risk) but also with a view to longer term agendas such as participant benefit. The initial decision whether to act on information, store it for later or ignore it is fundamental. The need for ASC to make decisions regarding changing weather during a day-long coaching session and its impact on content, duration and venue in the immediate, short, mid and longer term provides a potent example of the dynamical challenge faced by the ASC. 
Simon (1956) considers that classic decision-making models are linear/rational processes that are devoid of emotion. Thompson and Dowding (2002) describe this rational part as normative, assuming that the decision maker is being logical, rational and acting in an ideal world. Under these approaches, the quality of the decision relates directly to outcome. More recently, however, naturalistic decision making (NDM-Klein, 2008; Zsambok \& Klein, 1997) approaches have offered a more parsimonious account for coach decision making, especially in-event, where decisions are often high stakes and time pressured. Working from this NDM perspective, Cioffi and Markham (1997) recognize the part that emotions play and consider this in relation to intuitions. Such 'gut feel' decision-making has a long tradition in coaching (cf. Nash \& Collins, 2006) and even more overtly in the case of the ASC.

In this regard, Thompson and Dowding (2002) acknowledge intuition as a component of expert practice but also recognize a lack of consensus on the meaning of the term: English (1993) reports that intuitions are questionable, while Effken (2001) suggest they are subjective, immeasurable and un-teachable. It is our contention that intuition may be difficult to quantify and therefore to teach and learn but they are not un-teachable. Clearly, someone's intuitive drives have evolved from somewhere and, almost by definition therefore, must at some earlier stage have been conscious and rationalized. From a theoretical perspective, intuitive decisions equally appear to relate to a heuristics approach to decision making (Breakwell, 2007) and are particularly pertinent to the ASC given the frequency of self-reported, 'feels right' decision making (McCammon, 2004). In this regard, Stanovichand West (2000) propose a dual process in which decision making may have both emotive and logical parts; a structure that may explain McCammon's 'feel' right reports. Thus, yet another complicating factor comes into the risk-benefit decision, namely the coach's inclination towards making this 'feels' right decisions. The weaknesses of these intuitive approaches are clearly outlined in avalanche prediction and risk management decisions by McCammon (2004). He proposed that six heuristic 'traps' can compromise the decision making process in an outdoor context: familiarity with the context of the decision (familiarity with venue), consistency and alignment with other decisions (a fixed plan), acceptance of the levels of risk associated with making the decision ('it's my job to make hard calls'), a false sense of security or heightened expectation as the expert 
halo ('I'm the expert and always make good calls'), social impacts in the decision and scarcity of the conditions for the activity (it's a powder day!). Galloway (2005) suggests 'satisficing,' acceptance of the first workable option, and 'de-minimus,' emotional attachment to the goal, as further traps while Clements (1997) suggests ignorance of the context and Stemba (2005) the positive outcome, 'it can't happen to me' trap. All these traps have significant impact in 'marginal' decisions, in which the risk level and likelihood of incidence is both moderate to high, namely the most challenging! Of course, it must be acknowledged that the ability to make such judgments with apparent ease and success appears highly regarded, at least in the sports coaching world where such things have been evaluated (Abraham \& Collins, 2011). Yet again, the need for more applied research is highlighted so that this highly regarded skill may be optimally developed.

Specifically, such research must examine the process by which a skillful decision maker reaches a judgment in dynamic environments such as the outdoors. Kahneman and Klien (2009) consider heuristic and NDM processes. Both approaches have significant areas of commonality. For example, the traps highlighted earlier have the potential to affect both heuristic and naturalistic processes, especially given the personal nature and degree of impact on the individual. The degree of each trap's impact appears to depend on the situation, the desired outcome, the 'validity of the experience,' the role/position within the group and the personality of the decision maker. Accordingly, it is appropriate to consider these traps in the outdoor context as significant but to differing extents, based on the breadth and depth of the decision maker's experience, confidence, position in the group, understanding of the process and context. Finally, it should be recognized that McCammon's research focused on informally led groups. Accordingly, a refocusing on the professional judgment and decision making process of more formally led groups may lead to adaptation of the traps or discovery of new ones to reflect professional pressures.

Against the backdrop of these potential pitfalls, an expert decision maker will need be aware of the shortcomings in different approaches and able to monitor and modify style accordingly. Thus, the decision maker's ability to recognize situations/clues and commonalities in which specific processes may or may not be appropriate would suggest an ongoing attention to factors in both environment and participants. It is logical to consider that, in this context, these decisions may well be 
both heuristic and naturalistic to some extent. Accordingly, the knowledge required to recognize the values and weakness of both (and adjust accordingly) could be considered a meta-judgment, in that an evaluation (decision) about how these factors are changing and what this means precedes a second decision about what to do. This will directly relate to how the coach has 'constructed' the decision making model which, in turn, will be related to how experience is gained and reflected on and the nature of that knowledge. Supporting this chain of reasoning, Martin et al. (2006) recognize reflection as key to developing judgment.

Awareness, self-management and appraisal are key elements of meta-judgment in practice. As such, they are important features of coach development that, we suggest, may currently not receive sufficient (or sufficiently formal) attention in training, certification and on-going professional development, particularly in ASCs. This concern notwithstanding, it appears that the components of meta-judgment practice are congruent with reflective activity; this reflective process making explicit what may be originally tacit (Polyani, 1966; Nash \& Collins, 2006), enabling post hoc rationalizations of action taken in the 'heat of the moment.'

\section{Training to Realize the Benefits: Reflection in Professional Judgment and Decision Making}

Explicit comprehension of meta-judgment appears to form an important part of the professional judgment and decision-making (PJDM) repertoire for the ASC (cf. Martindale \& Collins, 2010; Abraham \& Collins, 2011). This involves explicit reflection in order to identify the declarative knowledge required to support and evolve that process. In this context, these meta-judgments may remain both intuitive and analytical in nature, exploiting both tacit and explicit knowledge bases in tandem.

Accordingly, the decision making abilities of an outdoor professional rely on a synergy of offline activity, including knowledge, experience, and self-reflection, as well as the more usually expected (and logged) practical time served on the hill/sea/river. The sub-conscious 'construction' of tacit knowledge acts to interpret and simplify complex information and appears to support a heuristic view (Guthrie, 1996) while creating explicit knowledge groups and relating variables together supports a more naturalistic process. It also seems likely that these synergetic processes sit within a 
nested decision making process (Abraham \& Collins, 2011). Interestingly, Nonaka (2009) suggests that these interactions are characterized as occurring in various environments; tacit to tacit exchange in a social context, explicit to explicit, in academic contexts, explicit to tacit and - tacit to explicit—in 'pracademic' contexts via some grounded theory and action-based research in which practitioner assumptions and experiences are challenged and explored through reflective practice. If this were true, then it would be interesting to assay the extent to which all four could play a part in ASC development systems.

The breakthrough in understanding decision making in relation to risk management will occur when there is mobilization of that knowledge; in other words, we carefully monitor what happens in practice against what 'experts' say they want to achieve. Tracking the long-term changes in coaches' thinking and behavior as a result of training and development activities will be another important step. Tacit knowledge often forms the basis of practitioner judgment. Investigation in the field to make explicit the decision making process will directly inform the tuition of decision making skills in ASC education, reflective practice being an essential element in that process and contributing to PJDM.

\section{Reflection in PJDM}

Anecdotally, good judgment is linked to experience and it is this idea that, at first glance, superficially supports a heuristic approach. However, Martin et al. (2006) stress the significance of reflection in the development of good judgment. The simplistic notion that good judgment comes from experience and that experience comes from bad judgment fails to recognize the value of reflection. It appears sensible to conclude that effective reflection on previous 'bad judgments' would, if appropriate in style and impact, support both professional and meta-judgment in future decision making for the ASC. Decision-making in this context is very complex, however, and is a synergy of processes facilitated by reflection. The explicit interaction (Polyani, 1962) of the analytic and intuitive elements acts as a 'force multiplier' and is as critical as the individual parts: the sum being greater than the value of the component parts. A synergetic relationship between the emotive and logical parts of dual process theory (Stanovich \& West, 2000) is, therefore, the desired outcome of any ASC development process. 
Reflection in this context needs to take place both on and in action (Schön, 1983), especially given the time periods and environments associated with adventure activities and the nested nature of the process. AS are not necessarily riskier in absolute terms than other activities. Rather, it is the nature of the risk, being omnipresent and ultimately uncontrollable, that characterizes the activity. This prevents 'stop and think' approaches espoused in more 'conventional' sports (cf. Schön, 1983). Accordingly, the reflective process must be positioned within the changing environment, linking the environment and decisions drawing on prior experience in that environment and situation while anticipating the future decisions and events that may develop. We suggest that multilevel, nested reflection is integral to nested decision making.

Yet again, a wide variety of research questions emerge. Do ASCs create time for reflection/decision-making? Is this ad-hoc, taking opportunity when it arises or rather, is it a considered aspect of the coaching process? Namely is opportunity created in the process? Further research into this practical aspect of the decision making process will be required in order to establish the tacit or explicit natures of the decision making process on ASC practice.

\section{Conclusion}

Enhanced risk benefit analysis is a distinct and crucial process for the ASC; a focus on the benefits, rather than on the elimination of risk is fundamental and matched with a deep comprehension of the individual response to risk. It is clear that increasing the level of risk will not automatically equate to better learning (Wolf \& Samdahl, 2005) and that educational objectives may be better met by changes to the pedagogic elements of the process (Brown \& Fraser, 2009). However, ASCs while working to develop a technical performance may adopt a process of hazard identification, risk assessment and manipulation of the perceived and real dangers in order to maximize potential benefit is underpinned by a complex decision making process in which emphasis must be placed on the positive and negative aspects associated with risk taking if balanced against any perceived benefit. Given the current 'risk averse' culture, understanding and explaining the benefits is a key precursor to the decision making process if judgments are to fit in with others' perceptions of what is acceptable. This is, at present, a feature from a legislative sense in which peer opinions form a crucial part of 
criminal court case against malpractice and/or negligence, including inappropriate risk and decision making!

In summary, ASC decision-making is not without its own inherent risks, a critical part of this process is the ability to recognize the benefits and that this relies on the 'expertise' in decision-making ability of the ASC. This, in turn, has a direct implication on the training and accreditation of ASCs. Making decisions based on the characteristics and styles of acknowledged experts (rather than just copying their decisions!) will facilitate better judgment at all levels. As McCammon (2004) identified, the tuition of judgment and decision-making appears crucial yet is conspicuous in its absence from most ASC, guide, and outdoor educational and governing body programs. Effective decision-making is the 'glue' that binds the skill of the ASC as, we suggest, is also the case for the sports coach, outdoor leader and educator. Therefore, to create the adaptive expertise required, attention must be paid to the decision-making skills of the individuals involved if we are to ensure effective, safe and good quality coaching practice.

Of course, these conclusions all require further research and investigation in a manner that reflects the pracademic reality of decision making in relation to risk exploitation in ASC. We are currently working to create an empirical base through a process of collaborative research with academic and practitioners. This mixed method approach will enable us to identify and design a model for professional judgment and decision making in adventure sports and outdoor education that has both a practical and academic credibility. It is hoped that this will further inform the debate which we hope this paper will stimulate. 


\section{References}

Abraham, A., \& Collins, D. (2011). Taking the next step: Ways forwards for coaching science. Quest, 63(4), 366-384.

Ball, D., Gill, T., \& Spiegel, B. (2008). Managing risk in play provision: Implementation guide. Annesley, DCSF publications.

Breakwell, G. M. (2007). The psychology of risk. Cambridge: Cambridge University Press.

Brevik, G. (2007). The quest for excitement and the safe society. In: M. J. McNamee (Ed.), Philosophy, risk and adventure sport. Abingdon: Routledge.

Brown, M., \& Fraser, D. (2009). Re-evaluating risk and exploring educational alternatives. Journal of Adventure Education and Outdoor Learning, 9(1), 61-77.

Brymer, E., \& Gray, T. (2010). Dancing with nature: Rhythm and harmony in extreme sport participation. Journal of Adventure Education and Outdoor Learning, 9(2), 135-149.

Chambers, P., Odeggard, E. E., \& Rinaldi, E. (2007). Risk taking and reflective learning. Reflective Practice, $8(2), 163-176$.

Cioffi, J., \& Markham, R. (1997). Clinical decision making by midwives: Managing complexity. Journal of Advanced Nursing, 25(2), 265-272.

Clements, K. (1997). The psychology of judgement for Outdoor Leaders. Presented at the International Conference on Outdoor Recreation and Education. Retrieved at:http://eric.ed.gov/ PDFS/ed417047.pdf

Collins, L., \& Collins, D. (2012). Contextualising the adventure sport coach. Journal of Adventure Education and Outdoor Learning, 10(1), 81-93.

Cross, N. (1999). Individualization of training programmes. In N. Cross and J. Lyle (Eds.) The coaching process: Principles and practice for sport. Oxford: Butterworth Heinemann, pp. 174-191.

Csikszentmihalyi, M. (1990). Flow: The psychology of optimal experience. New York: Harper and Row.

Effken, J. A. (2001). Nursing theory and concept development and analysis. Journal of Advanced Nursing, 34(2), 246-260.

English, I. (1993). Intuition as a function of the expert nurse: A critique of Benner's novice to expert model. Journal of Advanced Nursing, 18(3), 387-393.

Galloway, S. (2005). Judgement and decision-making In Outdoor leadership: Critical factors, common missteps, and keys to success. Proceedings of the 2005 National Conference on Outdoor Leadership. 
Gassner, M. E., \& Russel, K. C. (2008). Relative impact of course components at Outward Bound Singapore: A retrospective study of long term outcomes. Journal of Adventure Education \& Outdoor Learning, 8(2), $133-156$.

Gill, T. (2010). Nothing ventured. English Outdoor Council: FSC publications.

Hattie, J., Marsh, H. J., Neill, J., \& Richards, G. (1997). Adventure education and Outward Bound: Out of Class experiences that make a lasting difference. Review of Educational Research, 67(1), 43-87.

Health and Safety Executive. (2006). 5 steps to Risk Assessment. Retrieved at:www.hse.gov.uk/pubns/indg163.pdf

Hunt, J. (1990). Ethical issues in experiential education. Boulder, CO: Kendal/Hunt Publishing Company.

Kahneman, D., \& Klien, G. (2009). Conditions for Intuitive Expertise, a failure to disagree. American Psychologist, 64(6), 515-526.

Klein, G. (2008). Naturalistic decision making. Human Factors, 50(3), 456-460.

Lupton, D., \& Tulloch, J. (2002) 'Life would be pretty dull without risks,' Voluntary risk-taking and its pleasures. Health Risk and Society, 4(2), 113-124.

Lyle, J., \& Cushion, C. (2010). Sports coaching: Professionalisation and practice. Oxford: Churchill Livingston.

McCammon, I. (2004). Heuristic traps in recreational avalanche accidents: Evidence and implications. Avalanche News, 68, 1-10.

McDaniels T., \& Small M. (2004). Risk analysis and society. Cambridge: Cambridge University Press.

Martindale, A., \& Collins, D. (2010). But why does what works work? A response to Fifer, Henschen, Gould, and Ravizza. The Sport Psychologist, 24(1), 113-116.

Martin, B., Cashel, C., Wagstaff, M., \& Breunig, M., (2006). Outdoor leadership. Theory and practice. Champaign, IL: Human Kinetics.

Moxnes, P. (1989) Hverdagen angst I individ, gruppe og organisation, Oslo; Forlaget Paul Moxnes as cited in Brevik, G. (2007). The quest for excitement and the safe society. In: M. J. McNamee (Ed.), Philosophy, risk and adventure sport. Abingdon: Routledge.

Nash, C., \& Collins, D. (2006). Tacit knowledge in expert coaching: Science or art? Quest, 58(4), 465-477.

Nonaka, I. (2009). Tacit knowledge and knowledge Conversion: Controversy and advancement in organizational knowledge creation theory. Organization Science, 20(3), 635-652.

Polanyi, M. (1966). The tacit dimension. London: The University of Chicago Press. 
Priest, S., \& Gass, M. A. (2005). Effective leadership in adventure programming. Leeds: Human Kinetics

Simon, H. A. (1956). Rational choice and the structure of the environment. Psychological Review, 63(2), 129138.

Schön, D. (1983). The reflective practitioner: How professionals think in action. Aldershot: Ashgate.

Stanovich, K.E. \& West, R.F. (2000). Individual differences in reasoning: Implications for the rationality debate. Behavioural \& Brain Sciences, 23, 645-665.

Stemba, B. (2005). Traps and tips of decision making for outdoor leaders. Proceedings of the 2005 National Conference on Outdoor Leadership.

Tovey, H. (2007). Playing outdoors, spaces and places, risk and challenge, Maidenhead: Open University Press.

Thompson, C., \& Dowding, D. (2002). Decision making and judgment in nursing — an introduction. London: Churchill Livingston.

Ward, W. (2008). Perceptions of Risk and Expected Benefits of Users on Mount Whitney. PhD Thesis, Indiana University [Online]. Available at: http://gradworks.umi.com/33/19/3319896.html

Wilson, R., \& Crouch, E. A. C. (2001). Risk-benefit analysis. New York: Harvard University Press.

Wolf, B., \& Samdahl, D. (2005). Challenging assumptions: Examining fundamental beliefs that shape challenge course programming and research. Journal of Experiential Education, 28(1), 25-43.

Wurdinger, S. D. (1997). Philosophical Issues in Adventure Education (3 ${ }^{\text {rd }}$ Ed.). Boulder, CO: Kendal/Hunt. Young. (2010). Common sense Common Safety. Retrieved from lordyoungreview@dwp.gsi.gov.uk Zsambok, C. E., \& Klein, G. (Eds.). (1997). Naturalistic decision making expertise. Mahwah, NJ: Erlbaum. 
Note

1. The culture in which an individual is focused on attempts to gain compensation for any suffering, loss, or damage caused by another individual or organization. 